\title{
Ordering groups and validity in lattice-ordered groups
}

\author{
Almudena Colacito ${ }^{1}$ \\ Mathematical Institute, University of Bern, Sidlerstrasse 5, 3012 Bern, Switzerland \\ George Metcalfe ${ }^{1, *}$ \\ Mathematical Institute, University of Bern, Sidlerstrasse 5, 3012 Bern, Switzerland
}

\begin{abstract}
An inductive characterization is given of the subsets of a group that extend to the positive cone of a right order on the group. This characterization is used to relate validity of equations in lattice-ordered groups ( $\ell$-groups) to subsets of free groups that extend to the positive cone of a right order. As a consequence, new proofs are obtained of the decidability of the word problem for free $\ell$-groups and generation of the variety of $\ell$-groups by the $\ell$-group of automorphisms of the real line. An inductive characterization is also given of the subsets of a group that extend to the positive cone of an order on the group. In this case, the characterization is used to relate validity of equations in varieties of representable $\ell$-groups to subsets of relatively free groups that extend to the positive cone of an order.
\end{abstract}

Keywords: Ordered groups, Free groups, Lattice-ordered groups, Decidability 2000 MSC: 03B25, 03C05, 06F05, 06F15, 20E05

\section{Introduction}

The first aim of this paper is to establish a correspondence between validity of equations in lattice-ordered groups ( $\ell$-groups) and subsets of free groups that extend to positive cones of right orders on the group, thereby relating validity in

\footnotetext{
${ }^{*}$ Corresponding author

Email addresses: almudena.colacito@math.unibe.ch (Almudena Colacito), george.metcalfe@math. unibe.ch (George Metcalfe)

${ }^{1}$ Supported by Swiss National Science Foundation grant 200021_165850 and the EU Horizon 2020 research and innovation programme under the Marie Skłodowska-Curie grant agreement No 689176.
} 
$\ell$-groups also to properties of the topological spaces of right orders on free groups. This correspondence is used to obtain new proofs of the decidability of the word problem for free $\ell$-groups and generation of the variety of $\ell$-groups by the $\ell$-group of automorphisms of the real line. A correspondence is also established between validity of equations in varieties of representable $\ell$-groups (equivalently, classes of ordered groups) and subsets of relatively free groups that extend to positive cones of orders on the group. Our main tools for proving these results will be ordering theorems for groups that stem from proof-theoretic investigations and require very little structure theory for ordered groups.

Recall first, referring to [18] for further details and references, that an $\ell$-group is an algebraic structure $\mathbf{L}=\left\langle L, \wedge, \vee, \cdot,{ }^{-1}, e\right\rangle$ such that $\left\langle L, \cdot,{ }^{-1}, e\right\rangle$ is a group and $\langle L, \wedge, \vee\rangle$ is a lattice with an order $a \leq b: \Leftrightarrow a \wedge b=a$ that is compatible with left and right multiplication (i.e., $a \leq b$ implies $c a d \leq c b d$ for all $a, b, c, d \in L$ ). If $\leq$ is also total, then $\mathbf{L}$ is called an ordered group (o-group). The class $\mathcal{L G}$ of $\ell$-groups forms a variety, and the class of o-groups generates the variety $\mathcal{R} \mathcal{G}$ of representable $\ell$-groups. By a fundamental theorem of Holland, every $\ell$-group embeds into the group Aut $(\langle\Omega, \leq\rangle)$ of order-preserving bijections of a totally ordered set $\langle\Omega, \leq\rangle$ equipped with coordinate-wise lattice operations [13]. This representation was used by Holland in [14] to prove that $\mathcal{L G}$ is generated as a variety by $\operatorname{Aut}(\langle\mathbb{R}, \leq\rangle)$ and, with McCleary in [15], to establish the decidability of the word problem for free $\ell$-groups. Alternative proofs of these theorems that avoid the use of Holland's embedding theorem and sharpen the decidability result to coNP completeness were given by Galatos and Metcalfe [10]; this approach forms part of a broader program that aims to develop relationships between $\ell$-groups and varieties of residuated lattices (see, e.g., [2, 11, 12]).

Recall next that a partial order $\leq$ on the universe of a group $\mathbf{G}=\left\langle G, \cdot,{ }^{-1}, e\right\rangle$ is called a partial right order on $\mathrm{G}$ if it is compatible with right multiplication (i.e., $a \leq b$ implies $a c \leq b c$ for all $a, b, c \in G$ ), and a right order if it is also total. The positive cone $P_{\leq}:=\{a \in G \mid e<a\}$ of a partial right order on $\mathbf{G}$ is always a subsemigroup of $\mathbf{G}$ (i.e., $a, b \in P_{\leq}$implies $a b \in P_{\leq}$) that omits $e$. Conversely, if $P \subseteq G$ is a subsemigroup of $\mathbf{G}$ omitting $e$, then $a \leq^{P} b: \Leftrightarrow b a^{-1} \in P \cup\{e\}$ defines a partial right order on $\mathrm{G}$ satisfying $P_{\leq P}=P$. Hence from now on, we will identify partial right orders on $\mathbf{G}$ with subsemigroups of $\mathbf{G}$ that omit $e$, and right orders with partial right orders $P$ such that $a \in P$ or $a^{-1} \in P$ for all $a \in G \backslash\{e\}$. In particular, each right order on $\mathrm{G}$ can be viewed as a subset of $G$ and the set $\mathcal{R O}(\mathbf{G})$ of right orders on $\mathrm{G}$ forms a compact totally disconnected topological space with the subspace topology inherited from the product topology on the powerset $2^{G}$. This topological perspective is explored in detail in $[21,3,8]$ 
and related to spectral spaces of $\ell$-groups in [5].

We prove here that a finite subset $\left\{t_{1}, \ldots, t_{n}\right\}$ of a free group $\mathbf{F}$ extends to a right order on $\mathbf{F}$ if and only if the inequation $e \leq t_{1} \vee \cdots \vee t_{n}$ fails in some $\ell$-group (Theorem 2). Since every $\ell$-group term is equivalent in $\mathcal{L G}$ to a term of the form $\bigwedge_{i \in I} \bigvee_{j \in J_{i}} t_{i j_{i}}$ where each $t_{i j_{i}}$ is a group term, this correspondence provides a full characterization of validity in $\mathcal{L G}$. The result may be established using Hollister's theorem [16] that the lattice order of an $\ell$-group is the intersection of right orders on its group reduct. However, we use here instead an inductive characterization of subsets of groups that extend to right orders (Theorem 1, closely related to a theorem of Conrad [7]), to obtain a proof that requires almost no structure theory of $\ell$-groups. We then make use of the correspondence to obtain new proofs of the generation of $\mathcal{L G}$ as a variety by $\operatorname{Aut}(\langle\mathbb{R}, \leq\rangle)$ and the decidability of the word problem for free $\ell$-groups, the latter by appealing to an algorithm by Clay and Smith that recognizes when a given finite subset of a finitely generated free group extends to a right order [4].

In the last part of the paper, we turn our attention to validity of equations in varieties of representable $\ell$-groups (equivalently, validity in classes of o-groups). A partial order on the universe of a group $\mathbf{G}=\left\langle G, \cdot,{ }^{-1}, e\right\rangle$ that is compatible with left and right multiplication is called a partial order on $\mathbf{G}$, and an order if it is total. Positive cones of partial orders on $\mathrm{G}$ then correspond to normal (i.e., closed under conjugation) subsemigroups of $\mathbf{G}$ omitting $e$. We give an inductive characterization of subsets of groups that extend to orders (Theorem 7, closely related to a theorem of Ohnishi [20]), and use this to prove that a finite subset $\left\{t_{1}, \ldots, t_{n}\right\}$ of a relatively free group of a variety $\mathcal{V}$ of groups extends to an order if and only if the inequation $e \leq t_{1} \vee \cdots \vee t_{n}$ fails in some o-group with group reduct in $\mathcal{V}$ (Theorem 8 ).

Let us remark finally that a proof-theoretic account of some of the results presented here is given in the conference paper [6]. Note also that Wessel in [22] develops an alternative syntactic approach to orderability of groups that yields a new proof of a finitary version of Sikora's theorem for free abelian groups [21].

\section{A right ordering condition for groups}

Let us fix a group $\mathbf{G}=\left\langle G, \cdot,{ }^{-1}, e\right\rangle$ and denote the subsemigroup of $\mathbf{G}$ generated by $S \subseteq G$ by $\langle S\rangle$. Clearly, $\langle S\rangle$ is a partial right order on $\mathbf{G}$ if and only if $e \notin\langle S\rangle$. The following characterization of the subsets of $G$ that extend to right orders on G is proved by a straightforward application of Zorn's lemma (see [1]): 
$(\dagger) S \subseteq G$ extends to a right order on $\mathrm{G}$ if and only if for all $a_{1}, \ldots, a_{n} \in G \backslash\{e\}$, there exist $\delta_{1}, \ldots, \delta_{n} \in\{-1,1\}$ satisfying $e \notin\left\langle S \cup\left\{a_{1}^{\delta_{1}}, \ldots, a_{n}^{\delta_{n}}\right\}\right\rangle$.

We make use here, however, of an alternative inductive description of these sets (similar to characterizations by Ohnishi [20] for orderable groups, and Conrad [7] for right-orderable groups) that is more suitable for establishing relationships with validity in $\ell$-groups. We define inductively for $n \in \mathbb{N}$ :

$$
\begin{aligned}
\mathrm{R}_{0}(\mathbf{G}) & =\left\{S \subseteq G \mid S \cap S^{-1} \neq \emptyset\right\} ; \\
\mathrm{R}_{n+1}(\mathbf{G}) & =\mathrm{R}_{n}(\mathbf{G}) \cup\left\{T \cup\{a b\} \mid T \cup\{a\}, T \cup\{b\} \in \mathrm{R}_{n}(\mathbf{G})\right\} ; \\
\mathrm{R}(\mathbf{G}) & =\bigcup_{n \in \mathbb{N}} \mathrm{R}_{n}(\mathbf{G}) .
\end{aligned}
$$

It follows that $\mathrm{R}(\mathbf{G}) \subseteq \mathcal{P}(G)$ is the smallest set containing $\mathrm{R}_{0}(\mathbf{G})$ such that $T \cup$ $\{a\}, T \cup\{b\} \in \mathrm{R}(\mathbf{G})$ implies $T \cup\{a b\} \in \mathrm{R}(\mathbf{G})$. Since any $S \subseteq G$ occurring in $\mathrm{R}(\mathbf{G})$ must occur in $\mathrm{R}_{n}(\mathbf{G})$ for some $n \in \mathbb{N}$, there exists in this case a finite binary tree of subsets of $G$ with root $S$ and leaves in $\mathrm{R}_{0}(\mathbf{G})$ such that each non-leaf node is of the form $T \cup\{a b\}$ and has parent nodes $T \cup\{a\}, T \cup\{b\}$.

Example 1. Let $\mathbf{Z}$ be the additive group of the integers. Then $\{1,-1\} \in \mathrm{R}_{0}(\mathbf{Z})$, so $\{1,-2\} \in \mathrm{R}_{1}(\mathbf{Z})$. But $\{2,-2\} \in \mathrm{R}_{1}(\mathbf{Z})$, so $\{3,-2\} \in \mathrm{R}_{2}(\mathbf{Z})$, and since also $\{3,-3\} \in \mathrm{R}_{2}(\mathbf{Z})$, it follows that $\{3,-5\} \in \mathrm{R}_{3}(\mathbf{Z}) \subseteq \mathrm{R}(\mathbf{Z})$. This chain of reasoning can be displayed as a binary tree of finite sets of integers as follows:

$$
\frac{\overline{\{3,-3\}} \frac{\overline{\{2,-2\}} \frac{\overline{\{1,-1\}}}{\{1,-2\}}}{\{3,-2\}}}{\{3,-5\}}
$$

It is easily proved that $S \subseteq \mathbb{Z}$ is in $\mathrm{R}(\mathbf{Z})$ if and only if $S$ contains elements $m \leq 0$ and $n \geq 0$. This corresponds to the fact that $S \subseteq \mathbb{Z}$ extends to a (right) order on $\mathbf{Z}$ (of which there are just two, the standard one and its dual) if and only if it contains only strictly positive elements or only strictly negative elements (see Theorem 1).

Example 2. Let $\mathbf{F}(2)$ denote the free group on two generators $x, y$. The following binary tree of subsets of $F(2)$ demonstrates that $\left\{x x, y y, x^{-1} y^{-1}\right\} \in \mathrm{R}(\mathbf{F}(2))$ :

$$
\frac{\overline{\left\{x, y y, x^{-1}\right\}} \quad \overline{\left\{x, y y, x^{-1}\right\}}}{\frac{\left\{x x, y y, x^{-1}\right\}}{\left\{x x, y y, x^{-1} y^{-1}\right\}}} \frac{\overline{\left\{x x, y, y^{-1}\right\}} \overline{\left\{x x, y, y^{-1}\right\}}}{\left\{x x, y y, y^{-1}\right\}}
$$


This corresponds to the fact that $\left\{x x, y y, x^{-1} y^{-1}\right\}$ does not extend to a right order on $\mathbf{F}(2)$ (see Theorem 1) and also the fact that the inequation $e \leq x x \vee y y \vee x^{-1} y^{-1}$ is valid in all $\ell$-groups (see Theorem 2 ).

The remainder of this section is devoted to proving the following result.

\section{Theorem 1.}

(a) A group $\mathbf{G}$ is right-orderable if and only if $\{a\} \notin \mathrm{R}(\mathbf{G})$ for all $a \in G \backslash\{e\}$.

(b) If a group $\mathrm{G}$ is right-orderable, then $S \subseteq G$ extends to a right order on $\mathrm{G}$ if and only if $S \notin \mathrm{R}(\mathbf{G})$.

We first establish some elementary properties of $\mathrm{R}(\mathbf{G})$ for an arbitrary group $\mathrm{G}$.

Lemma 1. For any $S \cup T \cup\{a, b\} \subseteq G$ :

(a) $S \cup\{e\} \in \mathrm{R}(\mathbf{G})$;

(b) if $S \in \mathrm{R}(\mathbf{G})$, then $S \cup T \in \mathrm{R}(\mathbf{G})$;

(c) if $S \in \mathrm{R}(\mathbf{G})$, then $S^{\prime} \in \mathrm{R}(\mathbf{G})$ for some finite $S^{\prime} \subseteq S$;

(d) if $S \cup\{a b\} \in \mathrm{R}(\mathbf{G})$, then $S \cup\{a, b\} \in \mathrm{R}(\mathbf{G})$;

(e) $S \in \mathrm{R}(\mathbf{G})$ if and only if $\langle S\rangle \in \mathrm{R}(\mathbf{G})$.

Proof. For (a), clearly $\left\{e, e^{-1}\right\}=\{e\} \subseteq S \cup\{e\}$ and, by definition, $S \cup\{e\} \in$ $\mathrm{R}(\mathbf{G})$. The claims in (b) and (c) follow by a straightforward induction on $k \in \mathbb{N}$ such that $S \in \mathrm{R}_{k}(\mathbf{G})$. For (d), observe that if $S \cup\{a b\} \in \mathrm{R}(\mathbf{G})$, then, by (b), $S \cup\{a, a b\} \in \mathrm{R}(\mathbf{G})$. But also $S \cup\left\{a, a^{-1}\right\} \in \mathrm{R}(\mathbf{G})$, and hence $S \cup\{a, b\} \in \mathrm{R}(\mathbf{G})$. Finally, for (e), the left-to-right-direction follows directly from (b), and the rightto-left-direction follows by applying (b) and (c) to obtain a finite $S^{\prime} \subseteq\langle S\rangle$ such that $S \cup S^{\prime} \in \mathrm{R}(\mathbf{G})$ and then applying (d) repeatedly to obtain $S \in \mathrm{R}(\mathbf{G})$.

We now prove the left-to-right direction of Theorem 1 part (b), noting that for this direction there is no need to assume the right orderability of $\mathbf{G}$.

Lemma 2. If $S \in \mathrm{R}(\mathbf{G})$, then $S$ does not extend to a right order on $\mathbf{G}$.

Proof. Using ( $\dagger$ ), it suffices to prove that for any $k \in \mathbb{N}$ and $S \in \mathrm{R}_{k}(\mathbf{G})$, there exist $c_{1}, \ldots, c_{n} \in G \backslash\{e\}$ such that for all $\delta_{1}, \ldots, \delta_{n} \in\{-1,1\}$,

$$
e \in\left\langle S \cup\left\{c_{1}^{\delta_{1}}, \ldots, c_{n}^{\delta_{n}}\right\}\right\rangle
$$

We prove this claim by induction on $k$. For the base case, if $S \in \mathrm{R}_{0}(\mathbf{G})$, then $\left\{a, a^{-1}\right\} \subseteq S$ for some $a \in G$, so $e=a a^{-1} \in\langle S\rangle$. For the inductive step, suppose 
that $S=T \cup\{a b\} \in \mathrm{R}_{k+1}(\mathbf{G})$ because $T \cup\{a\} \in \mathrm{R}_{k}(\mathbf{G})$ and $T \cup\{b\} \in \mathrm{R}_{k}(\mathbf{G})$. By the induction hypothesis twice, we may assume without loss of generality that there exist $c_{1}, \ldots, c_{n} \in G \backslash\{e\}$ such that for all $\delta_{1}, \ldots, \delta_{n} \in\{-1,1\}$,

$$
e \in\left\langle T \cup\left\{a, c_{1}^{\delta_{1}}, \ldots, c_{n}^{\delta_{n}}\right\}\right\rangle \quad \text { and } \quad e \in\left\langle T \cup\left\{b, c_{1}^{\delta_{1}}, \ldots, c_{n}^{\delta_{n}}\right\}\right\rangle .
$$

But then for all $\delta_{1}, \ldots, \delta_{n}, \delta_{n+1} \in\{-1,1\}$, we obtain as required

$$
e \in\left\langle T \cup\left\{a b, c_{1}^{\delta_{1}}, \ldots, c_{n}^{\delta_{n}}, a^{\delta_{n+1}}\right\}\right\rangle .
$$

To establish part (a) and the right-to-left direction of part (b) of Theorem 1, we prove two preparatory lemmas, related to Theorems 2.2 and 2.3 of [7].

Lemma 3. For any $S \subseteq G$ such that $S \notin \mathrm{R}(\mathbf{G})$, there exists a subsemigroup $T$ of $\mathbf{G}$ extending $S$ such that $T \notin \mathrm{R}(\mathbf{G})$, and $G \backslash T$ is a subsemigroup of $\mathbf{G}$.

Proof. Suppose that $S \notin \mathrm{R}(\mathbf{G})$ and consider the set $\mathcal{U}$ of all subsemigroups of G extending $S$ that are not contained in $\mathrm{R}(\mathbf{G})$, partially ordered by inclusion. Clearly $\langle S\rangle \in \mathcal{U}$ by part (e) of Lemma 1 . Moreover, if $\left(T_{i}\right)_{i \in I}$ is a chain in $\mathcal{U}$, then also $\bigcup_{i \in I} T_{i} \in \mathcal{U}$; otherwise $\bigcup_{i \in I} T_{i} \in \mathrm{R}(\mathbf{G})$ and, using parts (b) and (c) of Lemma 1, we would have $T_{i} \in \mathrm{R}(\mathbf{G})$ for some $i \in I$, a contradiction. Hence an application of Zorn's lemma yields a maximal element $T$ of $\mathcal{U}$.

To prove that $G \backslash T$ is a subsemigroup of $\mathbf{G}$, suppose that $b, c \in G \backslash T$. Then $T \cup\{b\}$ and $T \cup\{c\}$ both properly extend $T$. Since $T$ is maximal, it follows that $T \cup\{b\}, T \cup\{c\} \in \mathrm{R}(\mathbf{G})$. Hence also $T \cup\{b c\} \in \mathrm{R}(\mathbf{G})$ and, since $T \notin \mathrm{R}(\mathbf{G})$, we obtain $b c \in G \backslash T$.

Lemma 4. If $S \subseteq G$ satisfies $S \notin \mathrm{R}(\mathbf{G})$ and $\{a\} \notin \mathrm{R}(\mathbf{G})$ for all $a \in G \backslash\{e\}$, then $S$ extends to a right order on $\mathrm{G}$.

Proof. Suppose that $S \notin \mathrm{R}(\mathbf{G})$ and $\{a\} \notin \mathrm{R}(\mathbf{G})$ for all $a \in G \backslash\{e\}$. Consider the set $\mathcal{W}$ of all subsemigroups $T$ of $\mathbf{G}$ extending $S$ such that $e \notin T$ and $G \backslash T$ is a subsemigroup of $\mathrm{G}$, partially ordered by inclusion. It follows from Lemma 3 that $\mathcal{W}$ is non-empty. Moreover, if $\left(T_{i}\right)_{i \in I}$ is a chain in $\mathcal{W}$, then also $\bigcup_{i \in I} T_{i} \in \mathcal{W}$. Hence an application of Zorn's lemma yields a maximal element $P$ of $\mathcal{W}$.

We claim that $P$ is a right order on $\mathbf{G}$ extending $S$. Suppose for a contradiction that there exists $a \in G \backslash\{e\}$ such that $a, a^{-1} \notin P$. By Lemma 3, the assumption $\{a\} \notin \mathrm{R}(\mathbf{G})$ yields a subsemigroup $T_{a}$ of $\mathbf{G}$ containing $a$ such that $T_{a} \notin \mathrm{R}(\mathbf{G})$ and $G \backslash T_{a}$ is a subsemigroup of $\mathbf{G}$. In particular, $e \notin T_{a}$. We claim that the maximality of $P$ is contradicted by

$$
P^{*}=P \cup\left\{b \in T_{a} \mid b, b^{-1} \notin P\right\} .
$$


Observe first that $P^{*}$ properly extends $P$ and does not contain $e$. It remains to prove that $P^{*}$ and $G \backslash P^{*}$ are subsemigroups of $\mathbf{G}$.

Consider first $b, c \in P^{*}$. If $b, c \in P$, then $b c \in P \subseteq P^{*}$. Also, if $b, c \in T_{a}$ and $b, b^{-1}, c, c^{-1} \notin P$, then $b c \in T_{a}$ (since $T_{a}$ is a subsemigroup) and $b c, c^{-1} b^{-1} \notin P$ (since $G \backslash P$ is a subsemigroup), so $b c \in P^{*}$. Suppose now that $c \in P$ and $b \in T_{a}$ is such that $b, b^{-1} \notin P$. Observe that $b^{-1} b c=c \in P$. Since $G \backslash P$ is a subsemigroup and $b^{-1} \notin P$, we must have $b c \in P \subseteq P^{*}$.

Now consider $b, c \notin P^{*}$. In particular, $b, c \notin P$, so $b c \notin P$. There are three cases. If $b, c \notin T_{a}$, then $b c \notin T_{a}$ and hence, $b c \notin P^{*}$. If $b, c \in T_{a}$, then, since $b, c \notin P^{*}$, we must have $b^{-1}, c^{-1} \in P$. So also $c^{-1} b^{-1} \in P$, and it follows that $b c \notin P^{*}$. Suppose finally, without loss of generality, that $b \in T_{a}$ and $c \notin T_{a}$. Since $b \in T_{a}, b \notin P$, and $b \notin P^{*}$, we must have $b^{-1} \in P$. Equivalently, $c c^{-1} b^{-1} \in P$, which, together with the fact that $c \notin P$, implies $c^{-1} b^{-1} \in P$. Hence $b c \notin P^{*}$.

Proof of Theorem 1. For (a), note first that if $\{a\} \notin \mathrm{R}(\mathbf{G})$ for all $a \in G \backslash\{e\}$, then an application of Lemma 4 with $S=\emptyset$ yields a right order on $\mathbf{G}$. For the converse direction, suppose that $\mathbf{G}$ is right-orderable and let $a \in G \backslash\{e\}$. Then $\{a\}$ extends to a right order on $\mathbf{G}$ and Lemma 2 yields $\{a\} \notin \mathrm{R}(\mathbf{G})$. Part (b) now follows immediately from Lemma 2 and Lemma 4.

\section{Right orders on free groups and the word problem for free $\ell$-groups}

In this section, we establish a correspondence between valid $\ell$-group equations and subsets of free groups that extend to right orders. We use this correspondence to obtain new proofs of the decidability of the word problem for free $\ell$-groups (first proved by Holland and McCleary [15]) and the equivalent problem of checking when a given finite subset of a finitely generated free group extends to a right order (first proved by Clay and Smith [4]). We also obtain a new proof of the generation of $\mathcal{L} \mathcal{G}$ as a variety by $\operatorname{Aut}(\langle\mathbb{R}, \leq\rangle)$ (first proved by Holland [14]).

Let $\mathbf{T}(X)$ and $\mathbf{T}^{\ell}(X)$ denote the term algebras over a set $X$ for the languages of groups and $\ell$-groups, respectively. A group term $t \in T(X)$ is said to be reduced if $t=e$ or $t=x_{1}^{\lambda_{1}} x_{2}^{\lambda_{2}} \cdots x_{n}^{\lambda_{n}}$ (associating to the left) with $x_{1}, \ldots, x_{n} \in X$ and $\lambda_{1}, \ldots, \lambda_{n} \in\{-1,1\}$ such that $t$ contains no occurrence of $x x^{-1}$ or $x^{-1} x$ for any $x \in X$. The reduced form of a group term $t \in T(X)$ is the reduced group term obtained from $t$ by associating to the left and cancelling occurrences of $x x^{-1}$ or $x^{-1} x$ for $x \in X$ as necessary. We define the free group $\mathbf{F}(X)$ over $X$ (writing $\mathbf{F}(k)$ when $|X|=k \in \mathbb{N}$ ) to be the set of all reduced group terms in $T(X)$ with the product of reduced group terms $s, t$ defined as the reduced form of st. We write $t$ to denote both a term in $T(X)$ and the reduced form of $t$ in $F(X)$. 
Theorem 2. The following are equivalent for $t_{1}, \ldots, t_{n} \in T(X)$ :

(1) $\left\{t_{1}, \ldots, t_{n}\right\}$ does not extend to a right order on $\mathbf{F}(X)$;

(2) $\left\{t_{1}, \ldots, t_{n}\right\} \in \mathrm{R}(\mathbf{F}(X))$;

(3) $\mathcal{L G} \models e \leq t_{1} \vee \cdots \vee t_{n}$.

Proof. The equivalence (1) $\Leftrightarrow$ (2) follows immediately from Theorem 1 and the fact that $\mathbf{F}(X)$ is right-orderable (see, e.g., [3]).

We prove (2) $\Rightarrow(3)$ by induction on $k \in \mathbb{N}$ such that $\left\{t_{1}, \ldots, t_{n}\right\} \in \mathrm{R}_{k}(\mathbf{F}(X))$. For the base case, suppose that $t_{i}=t_{j}{ }^{-1}$ in $\mathbf{F}(X)$ for some $i, j \in\{1, \ldots, n\}$. Then $\mathcal{L G} \models t_{i} \approx t_{j}{ }^{-1}$, so, since $e \leq x \vee x^{-1}$ is valid in all $\ell$-groups, also $\mathcal{L G} \models e \leq t_{1} \vee \cdots \vee t_{n}$. For the inductive step, suppose that $t_{n}=u v$ and $\left\{t_{1}, \ldots, t_{n-1}, u v\right\} \in \mathrm{R}_{k+1}(\mathbf{F}(X))$ because

$$
\left\{t_{1}, \ldots, t_{n-1}, u\right\} \in \mathrm{R}_{k}(\mathbf{F}(X)) \quad \text { and } \quad\left\{t_{1}, \ldots, t_{n-1}, v\right\} \in \mathrm{R}_{k}(\mathbf{F}(X)) .
$$

By the induction hypothesis twice,

$$
\mathcal{L} \mathcal{G} \models e \leq t_{1} \vee \cdots \vee t_{n-1} \vee u \quad \text { and } \quad \mathcal{L} \mathcal{G} \models e \leq t_{1} \vee \cdots \vee t_{n-1} \vee v
$$

The validity of the quasi-equation $(e \leq x \vee y) \&(e \leq x \vee z) \Rightarrow(e \leq x \vee y z)$ in all $\ell$-groups (see, e.g., [10, Lemma 3.3 (iv)]) then yields $\mathcal{L G}=e \leq t_{1} \vee \cdots \vee t_{n-1} \vee u v$.

We prove $(3) \Rightarrow(1)$ by contraposition. Suppose that $\left\{t_{1}, \ldots, t_{n}\right\}$ extends to a right order $\leq$ on $\mathbf{F}(X)$. Then $t_{1}, \ldots, t_{n}$ are all negative with respect to the dual right order $\leq^{\partial}$ on $\mathbf{F}(X)$. Let $\varphi$ be the homomorphism from $\mathbf{T}^{\ell}(X)$ to the $\ell$-group $\operatorname{Aut}\left(\left\langle F(X), \leq^{\partial}\right\rangle\right)$ with coordinate-wise lattice-ordering $\leq^{p}$, defined by mapping each $x \in X$ to the order-automorphism $\varphi(x): s \mapsto s x$. Then each $t \in T(x)$ is mapped to the order-automorphism $\varphi(t): s \mapsto s t$. In particular, $\varphi\left(t_{i}\right)(e)=t_{i}<^{\partial} e$ for each $i \in\{1, \ldots, n\}$, and hence, since $<^{\partial}$ is total, for some $j \in\{1, \ldots, n\}$,

$$
\varphi\left(t_{1} \vee \cdots \vee t_{n}\right)(e)=t_{j}<^{\partial} e .
$$

But $\varphi(e)(e)=e$, so in $\operatorname{Aut}\left(\left\langle F(X), \leq^{\partial}\right\rangle\right)$,

$$
\varphi(e) \not^{p} \varphi\left(t_{1} \vee \cdots \vee t_{n}\right) \text {. }
$$

Hence $\mathcal{L} \mathcal{G} \not \models e \leq t_{1} \vee \cdots \vee t_{n}$.

Since, by $(\dagger)$, a set of elements of a group extends to a right order if and only if each of its finite subsets extends to a right order, we obtain the following result. 
Corollary 1. $S \subseteq T(X)$ extends to a right order on $\mathbf{F}(X)$ if and only if $\mathcal{L} \forall \forall$ $e \leq t_{1} \vee \cdots \vee t_{n}$ for all $\left\{t_{1}, \ldots, t_{n}\right\} \subseteq S$.

Theorem 2 can be generalized to arbitrary right-orderable groups. Given a class $\mathcal{L}$ of $\ell$-groups and $\Sigma \cup\{s \approx t\} \subseteq\left(T^{\ell}(X)\right)^{2}$, let $\Sigma \models_{\mathcal{L}} s \approx t$ denote that for any $\mathbf{L} \in \mathcal{L}$ and homomorphism $\varphi: \mathbf{T}^{\ell}(X) \rightarrow \mathbf{L}$, whenever $\varphi\left(s^{\prime}\right)=\varphi\left(t^{\prime}\right)$ for all $s^{\prime} \approx t^{\prime} \in \Sigma$, also $\varphi(s)=\varphi(t)$. Recall also that a group presentation $\langle X \mid R\rangle$ describes the quotient of the free group $\mathbf{F}(X)$ by the normal subgroup generated by $R \subseteq T(X)$; for $t \in T(X)$, we let $\bar{t}$ denote the equivalence class of the corresponding reduced group term $t \in F(X)$ in this quotient.

Theorem 3. Suppose that $\mathbf{G}=\langle X \mid R\rangle$ is a right-orderable group. Then the following are equivalent for $t_{1}, \ldots, t_{n} \in T(X)$ :

(1) $\left\{\bar{t}_{1}, \ldots, \bar{t}_{n}\right\}$ does not extend to a right order on $\mathbf{G}$;

(2) $\left\{\bar{t}_{1}, \ldots, \bar{t}_{n}\right\} \in \mathrm{R}(\mathbf{G})$;

(3) $\{r \approx e \mid r \in R\} \models_{\mathcal{L G}} e \leq t_{1} \vee \cdots \vee t_{n}$.

Proof. The equivalence (1) $\Leftrightarrow(2)$ follows again immediately from Theorem 1 and the fact that $\mathrm{G}$ is assumed to be right-orderable. Also, we can prove (2) $\Rightarrow$ (3) by induction on $k \in \mathbb{N}$ such that $\left\{\bar{t}_{1}, \ldots, \bar{t}_{n}\right\} \in \mathrm{R}_{k}(\mathbf{G})$ following the same reasoning as in the proof of Theorem 2 .

The proof of $(3) \Rightarrow(1)$ generalizes the reasoning of Theorem 2. Suppose contrapositively that $\left\{\bar{t}_{1}, \ldots, \bar{t}_{n}\right\}$ extends to a right order $\leq$ on $\mathbf{G}$. Then $\bar{t}_{1}, \ldots, \bar{t}_{n}$ are all negative with respect to the dual right order $\leq^{\partial}$ on $\mathrm{G}$. Let $\varphi$ be the map evaluating each $x \in X$ by the order-automorphism $\varphi(x): \bar{s} \mapsto \overline{s x}$ in the $\ell$-group $\operatorname{Aut}\left(\left\langle G, \leq^{\partial}\right\rangle\right)$ with coordinate-wise lattice-ordering $\leq^{p}$. Then each $t \in T(x)$ is mapped to the order-automorphism $\varphi(t): \bar{s} \mapsto \overline{s t}$. Observe that for all $r \in R$,

$$
\varphi(r)(\bar{s})=\overline{s r}=\overline{s e}=\bar{s}=\varphi(e)(\bar{s}) .
$$

So $\varphi$ satisfies $\{r \approx e \mid r \in R\}$. But also $\varphi\left(t_{i}\right)(\bar{e})=\bar{t}_{i}<^{\partial} \bar{e}$ for each $i \in$ $\{1, \ldots, n\}$, and hence, since $<^{\partial}$ is total,

$$
\varphi\left(t_{1} \vee \cdots \vee t_{n}\right)(\bar{e})<^{\partial} \bar{e}=\varphi(e)(\bar{e}) .
$$

So $\varphi(e) \not^{p} \varphi\left(t_{1} \vee \cdots \vee t_{n}\right)$. Hence $\{r \approx e \mid r \in R\} \forall_{\mathcal{L G}} e \leq t_{1} \vee \ldots \vee t_{n}$.

Example 3. Consider the fundamental group $\mathbf{K}=\left\langle x, y \mid x y x^{-1} y\right\rangle$ of the Klein bottle. It is easily shown that $\left\{x y x^{-1} y \approx e\right\}=_{\mathcal{L G}} e \leq y^{-1} x^{-1} \vee x$ and hence, by the preceding theorem, $\left\{\overline{y^{-1} x^{-1}}, \bar{x}\right\}$ does not extend to a right order on $\mathbf{K}$. 
We devote the rest of this section to decidability and generation problems. First, we recall the following result established by Holland and McCleary in [15].

Theorem 4. The word problem for free $\ell$-groups is decidable.

The following decidability result is then an immediate consequence of Theorem 2.

Theorem 5. The problem of deciding if a finite subset of a free group extends to a right order is decidable.

Galatos and Metcalfe have proved that the word problem for free $\ell$-groups is coNP-complete [10], and it follows that the problem of deciding whether or not a finite subset of a free group extends to a right order is also in the complexity class co-NP. It is not known, however, if this latter problem is co-NP-complete.

We now use the results of the previous section to present a proof of Theorem 4 that does not appeal to further algebraic results such as the Holland embedding theorem. As a byproduct, we obtain also an alternative proof that $\mathcal{L G}$ is generated as a variety by $\operatorname{Aut}(\langle\mathbb{R}, \leq\rangle)$.

Given $S \subseteq T(X)$, let $S^{r}$ denote the corresponding set of reduced group terms. We define is $(S):=\left\{u \in T(X) \mid u v \in S^{r} ; u, v\right.$ reduced group terms $\}$ and $\operatorname{cis}(S):=\left\{u v^{-1} \mid u, v \in \operatorname{is}(S) ; u \neq v\right\}$.

Proposition 1. The following are equivalent for any $t_{1}, \ldots, t_{n} \in T(X)$ :

(1) $\mathcal{L} \mathcal{G} \models e \leq t_{1} \vee \cdots \vee t_{n}$;

(2) $\operatorname{Aut}(\langle\mathbb{R}, \leq\rangle) \models e \leq t_{1} \vee \cdots \vee t_{n}$;

(3) For $\left\{s_{1}, \ldots, s_{m}\right\}=\operatorname{cis}\left(\left\{t_{1}, \ldots, t_{n}\right\}\right)$ and all $\delta_{1}, \ldots, \delta_{m} \in\{-1,1\}$,

$$
e \in\left\langle\left\{t_{1}, \ldots, t_{n}, s_{1}^{\delta_{1}}, \ldots, s_{m}^{\delta_{m}}\right\}\right\rangle
$$

Proof. (1) $\Rightarrow(2)$ is immediate. For (3) $\Rightarrow(1)$, observe that (3) implies, using $(\dagger)$, that $\left\{t_{1}, \ldots, t_{n}\right\}$ does not extend to a right order on $\mathbf{F}(X)$, and hence, by Theorem 2, that $\mathcal{L G}=e \leq t_{1} \vee \cdots \vee t_{n}$.

$(2) \Rightarrow(3)$ is proved in some detail in [10]; we recall the main ingredients of this proof here for the sake of completeness. Suppose contrapositively that $e \notin\langle S\rangle$ for some choice of $\delta_{1}, \ldots, \delta_{m} \in\{-1,1\}$ and $S=\left\{t_{1}, \ldots, t_{n}, s_{1}^{\delta_{1}}, \ldots, s_{m}^{\delta_{m}}\right\}$. Let $a_{u}$ be a variable for each $u \in \operatorname{is}\left(\left\{t_{1}, \ldots, t_{n}\right\}\right)$. We define a set of inequations $T$ consisting of all $a_{u}<a_{v}$ such that $u, v \in$ is $\left(\left\{t_{1}, \ldots, t_{n}\right\}\right)$ and $u v^{-1} \in\langle S\rangle$.

Claim 1. $T$ is satisfiable over $\mathbb{R}$. 
Proof. Suppose for a contradiction that $T$ is unsatisfiable over $\mathbb{R}$, i.e., there is no assignment of real numbers to the variables that satisfies all the inequations in $T$ with respect to the standard order. Then there must exist a chain $a_{u_{1}}<$ $a_{u_{2}}<\ldots<a_{u_{k}}<a_{u_{1}}$ in $T$. But then $e=u_{1} u_{2}{ }^{-1}, u_{2} u_{3}{ }^{-1}, \ldots, u_{k} u_{1}^{-1} \in S$, contradicting our assumption.

By Claim 1, there exists a map sending each $a_{u}$ for $u \in$ is $\left(\left\{t_{1}, \ldots, t_{n}\right\}\right)$ to a real number $r_{u}$ that satisfies $T$. In particular, $t_{i} e^{-1} \in S$ for each $i \in\{1, \ldots, n\}$, and hence $a_{t_{i}}<a_{e} \in T$, yielding $r_{t_{i}}<r_{e}$. Now for each $x \in X$, define a partial map $\hat{x}$ from $\mathbb{R}$ to $\mathbb{R}$, that sends $r_{u}$ to $r_{u x}$ if $u, u x \in$ is $\left(\left\{t_{1}, \ldots, t_{n}\right\}\right)$, and $r_{u x^{-1}}$ to $r_{u}$ if $u x^{-1}, u \in$ is $\left(\left\{t_{1}, \ldots, t_{n}\right\}\right)$.

Claim 2. $\hat{x}$ is order preserving.

Proof. Suppose first that $\hat{x}$ maps $r_{u}$ to $r_{u x}$ and $r_{v}$ to $r_{v x}$, but $r_{u}<r_{v}$ and $r_{v x}<$ $r_{u x}$. Then $u v^{-1} \in S$ and $(v x)\left(x^{-1} u^{-1}\right)=v u^{-1} \in S$, so $a_{u}<a_{v} \in T$ and $a_{v}<a_{u} \in T$, contradicting Claim 1. Alternatively, suppose that $\hat{x}$ maps $r_{u}$ to $r_{u x}$ and $r_{v x^{-1}}$ to $r_{v}$, but $r_{u}<r_{v x^{-1}}$ and $r_{v}<r_{u x}$. Then $u\left(v x^{-1}\right)^{-1}=u x v^{-1} \in S$ and $v(u x)^{-1}=v x^{-1} u^{-1} \in S, a_{u x}<a_{v} \in T$ and $a_{v}<a_{u x} \in T$, contradicting Claim 1. Other cases are very similar.

Finally, we extend each $\hat{x}$ for $x \in X$ linearly to a function $\hat{\varphi}(x)$ in $\operatorname{Aut}(\langle\mathbb{R}, \leq\rangle)$. But then $\hat{\varphi}\left(t_{i}\right)\left(r_{e}\right)=r_{t_{i}}$ for each $i \in\{1, \ldots, n\}$, while $\hat{\varphi}(e)\left(r_{e}\right)=r_{e}$. Hence, $\hat{\varphi}\left(t_{1} \vee \cdots \vee t_{n}\right)\left(r_{e}\right)=r_{t_{j}}<r_{e}$ for some $j \in\{1, \ldots, n\}$. This establishes $\operatorname{Aut}(\langle\mathbb{R}, \leq\rangle) \forall=e \leq t_{1} \vee \cdots \vee t_{n}$ as required.

Implicit in the proof of this proposition is a decision procedure for checking the validity of an inequation $e \leq t_{1} \vee \cdots \vee t_{n}$ in $\mathcal{L G}$, and hence a proof of Theorem 4 . Namely, calculate $\operatorname{cis}\left(\left\{t_{1}, \ldots, t_{n}\right\}\right)$ and denote its elements by $s_{1}, \ldots, s_{m}$. For each choice of $\delta_{1}, \ldots, \delta_{m} \in\{-1,1\}$ and $S=\left\{t_{1}, \ldots, t_{n}, s_{1}^{\delta_{1}}, \ldots, s_{m}^{\delta_{m}}\right\}$, check the satisfiability of the resulting set of inequations $T$ over $\mathbb{R}$. If all of these sets are unsatisfiable, then we have established the equivalent condition (3) above, so $\mathcal{L G} \models e \leq t_{1} \vee \cdots \vee t_{n}$. Otherwise, there is a set of inequations $T$ that is satisfiable over $\mathbb{R}$ and, as described in the proof above, $\operatorname{Aut}(\langle\mathbb{R}, \leq\rangle) \forall e \leq t_{1} \vee \cdots \vee t_{n}$, so $\mathcal{L} \mathcal{G} \not \models e \leq t_{1} \vee \cdots \vee t_{n}$.

The equivalence of (1) and (2) also yields the following result, first proved by Holland [14].

Corollary 2. The variety $\mathcal{L G}$ of $\ell$-groups is generated by $\operatorname{Aut}(\langle\mathbb{R}, \leq\rangle)$.

This generation result can be interpreted in terms of extending subsets of free 
groups to right orders.

Corollary 3. A set of elements $\left\{t_{1}, \ldots, t_{n}\right\}$ of a free group over $x_{1}, \ldots, x_{k}$ extends to a right order if and only if there exist order-preserving bijections $\hat{x}_{1}, \ldots, \hat{x}_{k}$ of the real line such that $\hat{t}_{i}(0)<0$ for all $i \in\{1, \ldots, n\}$.

As mentioned above, Theorem 5 is a direct consequence of Theorems 2 and 4. However, an algorithm for recognizing when a finite subset of a finitely generated free algebra $\mathbf{F}(k)$ extends to a right order was already provided by Clay and Smith in [4]. Let $|t|$ denote the length of a reduced group term $t$ in $\mathbf{F}(k)$, and for $l \in \mathbb{N}$, let $F_{l}(k)=\{t \in F(k)|| t \mid \leq l\}$. Note that $F_{l}(k)$ is finite and can be viewed as the $l$-ball of the Cayley graph of $F(k)$ relative to $X$. For a subset $S$ of $\mathbf{F}(k)$ that omits $e$, we say that $S$ is an l-truncated right order on $\mathbf{F}(k)$ if $S=\langle S\rangle \cap F_{l}(k)$, and for all $t \in F_{l-1}(k) \backslash\{e\}$, either $t \in S$ or $t^{-1} \in S$.

Proposition 2 (Clay and Smith [4]). $S \subseteq F(k)$ extends to a right order on $\mathbf{F}(k)$ if and only if $S$ extends to an l-truncated right order on $\mathbf{F}(k)$ for some $l \in \mathbb{N}$.

Hence we obtain the following algorithm for deciding if a finite subset $S$ of $F(k)$ extends to a right order on $\mathbf{F}(k)$, noting that termination is guaranteed by the fact that $F_{l}(k)$ is finite for any $l \in \mathbb{N}$.

1. Let $l=\max \{|t| \mid t \in S\}$.

2. Let $M=\{S\}$.

3. If $M=\emptyset$, then output "no" and stop.

4. Select $S_{1} \in M$.

5. Construct $S_{1}^{*}=\left\langle S_{1}\right\rangle \cap F_{l}(k)$.

6. If $e \in S_{1}^{*}$, then remove $S_{1}$ from $M$ and go to 3 .

7. If $t \in S_{1}^{*}$ or $t^{-1} \in S_{1}^{*}$ for all $t \in F_{l-1}(k) \backslash\{e\}$, then output "yes" and stop.

8. Select $t \in F_{l-1}(k) \backslash\{e\}$ such that $t \notin S_{1}^{*}$ and $t^{-1} \notin S_{1}^{*}$.

9. Replace $S_{1}$ with $S_{1}^{*} \cup\{t\}$ and $S_{1}^{*} \cup\left\{t^{-1}\right\}$ in $M$ and go to 3 .

Example 4. Consider $S=\left\{x x, y y, x^{-1} y^{-1}\right\} \subseteq F(2)$. We let $l=2, M=\{S\}$ and select $S_{1}=S$ (steps 1-4). Adding all products in $F_{2}(2)$ of members of $S_{1}$ (step 5), we obtain

$$
S_{1}^{*}=\left\{x x, y y, x^{-1} y^{-1}, x y^{-1}, x^{-1} y, x y\right\} .
$$

Since $e \notin S_{1}^{*}, x \notin S_{1}^{*}, x^{-1} \notin S_{1}^{*}$ (steps 6-7), we select $x$ (step 8) and let $M=$ $\left\{S_{1}^{*} \cup\{x\}, S_{1}^{*} \cup\left\{x^{-1}\right\}\right\}$ (step 9). Selecting next $S_{2}=S_{1}^{*} \cup\{x\}$ (step 4) and taking 
products in $F_{2}(2)$ (step 5), we obtain a set $S_{2}^{*}$ that contains $x x^{-1} y x x^{-1} y^{-1}=e$. Hence we let $M=\left\{S_{1}^{*} \cup\left\{x^{-1}\right\}\right\}$ (step 6). Selecting now $S_{3}=S_{1}^{*} \cup\left\{x^{-1}\right\}$ (step 4) and taking products in $F_{2}(2)$ (step 5), we obtain a set $S_{3}^{*}$ that contains $x^{-1} x^{-1} x x=e$. Hence we let $M=\emptyset$ (step 6) and output "no" (step 3). It follows that $S$ does not extend to a right order on $\mathbf{F}(2)$ and

$$
\mathcal{L} \mathcal{G} \models e \leq x x \vee y y \vee x^{-1} y^{-1} .
$$

Consider now $T=\left\{x x, x y, y x^{-1}\right\} \subseteq F(2)$. We let $l=2, M=\{T\}$ and select $T_{1}=T$ (steps 1-4). Adding all products in $F_{2}(2)$ of members of $T_{1}$ (step 5), we obtain

$$
T_{1}^{*}=\left\{x x, x y, y x^{-1}, y x, y y\right\} .
$$

Since $e \notin T_{1}^{*}, x \notin T_{1}^{*}, x^{-1} \notin T_{1}^{*}$ (steps 6-7), we select $x$ (step 8) and let $M=$ $\left\{T_{1}^{*} \cup\{x\}, T_{1}^{*} \cup\left\{x^{-1}\right\}\right\}$ (step 9). Selecting $T_{2}=T_{1}^{*} \cup\{x\}$ (step 4) and taking products in $F_{2}(2)$ (step 5), we obtain

$$
T_{2}^{*}=\left\{x x, x y, y x^{-1}, y x, y y, x, y\right\} .
$$

Then $e \notin T_{2}^{*}$ (step 6) and $t \in T_{2}^{*}$ or $t^{-1} \in T_{2}^{*}$ for all $t \in F_{1}(k) \backslash\{e\}$ (i.e., $T_{2}^{*}$ is a 2 -truncated right order on $\mathbf{F}(2)$ ), so we output "yes" (step 7). Hence $T$ extends to a right order on $\mathbf{F}(2)$ and

$$
\mathcal{L G} \forall e \leq x x \vee x y \vee y x^{-1}
$$

We conclude this section by mentioning a topological result regarding right orders on free groups, and its interpretation in terms of validity in $\ell$-groups. Note first that a right order is isolated in the space $\mathcal{R O}(\mathbf{G})$ of right orders on a group $\mathrm{G}$ if and only if it is the unique right order extending some finite subset of $G$. In [19] McCleary proved the following result for spaces of right orders on finitely generated free groups.

Theorem 6 (McCleary [19]). $\mathcal{R O}(\mathbf{F}(k))$ has no isolated points for $k \geq 2$.

By Theorem 2, we obtain the following feature of validity in $\ell$-groups.

Corollary 4. The following are equivalent for $t_{1}, \ldots, t_{n} \in T(k)$ where $k \geq 2$ :

(1) $\mathcal{L} \mathcal{G} \models e \leq t_{1} \vee \cdots \vee t_{n}$;

(2) $\mathcal{L} \mathcal{G} \models e \leq t_{1} \vee \cdots \vee t_{n} \vee s$ or $\mathcal{L} \mathcal{G} \models e \leq t_{1} \vee \cdots \vee t_{n} \vee s^{-1}$ for all $s \in T(k)$. 
Proof. (1) $\Rightarrow(2)$ is immediate. For (2) $\Rightarrow$ (1), suppose that $\mathcal{L G} \not \models e \leq t_{1} \vee \cdots \vee t_{n}$. By Theorem 2, the set $\left\{t_{1}, \ldots, t_{n}\right\}$ extends to a right order on $\mathbf{F}(k)$. But also, by Theorem 6 , the space of right orders on $\mathbf{F}(k)$ has no isolated points, so there is more than one right order on $\mathbf{F}(k)$ extending $\left\{t_{1}, \ldots, t_{n}\right\}$. In particular, there must exist an $s \in T(k)$ such that both $\left\{t_{1}, \ldots, t_{n}, s\right\}$ and $\left\{t_{1}, \ldots, t_{n}, s^{-1}\right\}$ extend to right orders on $\mathbf{F}(k)$. Theorem 2 then yields $\mathcal{L G} \forall \forall e \leq t_{1} \vee \cdots \vee t_{n} \vee s$ and $\mathcal{L} \mathcal{G} \forall e \leq t_{1} \vee \cdots \vee t_{n} \vee s^{-1}$ as required.

Let us remark finally that Proposition 2 can be used to show that every isolated point of $\mathcal{R O}(\mathbf{F}(k))(k \geq 2)$ is finitely generated as a semigroup, which, together with a result of Kielak [17] that no right order of $\mathbf{F}(k)$ is finitely generated as a semigroup, yields an alternative proof of Theorem 6 (see [8] for details).

\section{Ordering relatively free groups and validity in ordered groups}

We turn our attention in this section to orders on relatively free groups and validity of equations in corresponding classes of ordered groups (equivalently, validity in varieties of representable $\ell$-groups). We begin by providing a characterization of subsets of a group that extend to orders. Since the results and proofs are very similar to those presented for right orders in Section 2, we confine ourselves here to presenting the main ingredients of the approach, pointing out only the most significant differences.

Let us again fix a group $\mathbf{G}=\left\langle G, \cdot,{ }^{-1}, e\right\rangle$ and denote by $\langle\langle S\rangle\rangle$ the normal subsemigroup of $\mathbf{G}$ generated by $S \subseteq G$. Clearly, $\langle\langle S\rangle\rangle$ is a partial order on $\mathbf{G}$ if and only if $e \notin\langle\langle S\rangle\rangle$. As in the case of right orders, the following characterization of subsets of $G$ that extend to orders on $\mathrm{G}$ is established by a straightforward application of Zorn's lemma (see [9]):

$(\ddagger) S \subseteq G$ extends to an order on $\mathbf{G}$ if and only if for all $a_{1}, \ldots, a_{n} \in G \backslash\{e\}$, there exist $\delta_{1}, \ldots, \delta_{n} \in\{-1,1\}$ such that $e \notin\left\langle\left\langle S \cup\left\{a_{1}^{\delta_{1}}, \ldots, a_{n}^{\delta_{n}}\right\}\right\rangle\right\rangle$.

Our alternative characterization (similar again to those obtained in [20] and [7]) supplements the characterization for right orders with an extra condition to take care of normality. We define inductively for $n \in \mathbb{N}$ :

$$
\begin{aligned}
& \mathrm{B}_{0}(\mathbf{G})=\left\{S \subseteq G \mid S \cap S^{-1} \neq \emptyset\right\} ; \\
& \mathrm{B}_{n+1}(\mathbf{G})=\mathrm{B}_{n}(\mathbf{G}) \cup\left\{T \cup\{a b\} \mid T \cup\{a\}, T \cup\{b\} \in \mathrm{B}_{n}(\mathbf{G})\right. \\
& \text { or } \left.T \cup\{b a\} \in \mathrm{B}_{n}(\mathbf{G})\right\} \text {; } \\
& \mathrm{B}(\mathbf{G})=\bigcup_{n \in \mathbb{N}} \mathrm{B}_{n}(\mathbf{G}) .
\end{aligned}
$$


It follows that $\mathrm{B}(\mathbf{G}) \subseteq \mathcal{P}(G)$ is the smallest set containing $\mathrm{B}_{0}(\mathbf{G})$ such that $T \cup\{a\}, T \cup\{b\} \in \mathrm{B}(\mathbf{G})$ implies $T \cup\{a b\} \in \mathrm{B}(\mathbf{G})$, and also $T \cup\{b a\} \in \mathrm{B}(\mathbf{G})$ implies $T \cup\{a b\} \in \mathrm{B}(\mathbf{G})$.

Example 5. In the free group $\mathbf{F}(2)$ generated by $x, y$, clearly $\left\{x, x^{-1}\right\} \in \mathrm{B}_{0}(\mathbf{F}(2))$ and hence $\left\{x, y x^{-1} y^{-1}\right\} \in \mathrm{B}_{1}(\mathbf{F}(2))$. This reflects the fact that $\left\{x, y x^{-1} y^{-1}\right\}$ does not extend to an order on $\mathbf{F}(2)$ and also the fact that $e \leq x \vee y x^{-1} y^{-1}$ is valid in all o-groups (see Theorem 8). Note, however, that $\left\{x, y x^{-1} y^{-1}\right\} \notin \mathrm{R}(\mathbf{F}(2)$ ), reflecting the fact that $\left\{x, y x^{-1} y^{-1}\right\}$ does extend to a right order on $\mathbf{F}(2)$ and the fact that $e \leq x \vee y x^{-1} y^{-1}$ is not valid in all $\ell$-groups (see Theorem 2).

Example 6. In the fundamental group $\mathbf{K}$ of the Klein bottle (see Example 3), $\left\{y, x y x^{-1}\right\} \in \mathrm{B}_{0}(\mathbf{K})$ and hence $\{y\} \in \mathrm{B}_{1}(\mathbf{K})$. This corresponds to the fact that $\mathbf{K}$ is not orderable (Theorem 7 ).

The proof of the following theorem proceeds similarly to the proof of Theorem 1, using condition $(\ddagger)$ to establish the left-to-right direction of (b), and considering normal subsemigroups of $\mathrm{G}$ extending $S \subseteq G$ to establish analogues of Lemmas 3 and 4.

\section{Theorem 7.}

(a) A group $\mathbf{G}$ is orderable if and only if $\{a\} \notin \mathrm{B}(\mathbf{G})$ for all $a \in G \backslash\{e\}$.

(b) If a group $\mathrm{G}$ is orderable, then $S \subseteq G$ extends to an order on $\mathrm{G}$ if and only if $S \notin \mathrm{B}(\mathbf{G})$.

We now establish a correspondence between subsets of relatively free groups that extend to orders, and the valid $\ell$-group equations of a corresponding class of ordered groups. Let $\mathcal{V}$ be a variety of groups and let $\mathcal{K}_{\mathcal{V}}$ be the class of all o-groups that have a group reduct in $\mathcal{V}$. In particular, if $\mathcal{V}$ is the variety of all groups, then $\mathcal{K}_{\mathcal{V}}$ is the class of all o-groups. We consider a relatively free group $\mathbf{F}_{\mathcal{V}}(X)$ of $\mathcal{V}$ over some non-empty set $X$ of generators, writing again $t$ to denote the element of $\mathbf{F}_{\mathcal{V}}(X)$ corresponding to a group term $t \in T(X)$.

Theorem 8. If $\mathbf{F}_{\mathcal{V}}(X)$ is orderable, then the following are equivalent for all $t_{1}, \ldots, t_{n} \in T(X)$ :

(1) $\left\{t_{1}, \ldots, t_{n}\right\}$ does not extend to an order on $\mathbf{F}_{\mathcal{V}}(X)$;

(2) $\left\{t_{1}, \ldots, t_{n}\right\} \in \mathrm{B}\left(\mathbf{F}_{\mathcal{V}}(X)\right)$;

(3) $\mathcal{K}_{\mathcal{V}} \models e \leq t_{1} \vee \cdots \vee t_{n}$. 
Proof. The equivalence (1) $\Leftrightarrow$ (2) follows immediately from Theorem 7 .

We prove $(2) \Rightarrow(3)$ by induction on $k \in \mathbb{N}$ such that $\left\{t_{1}, \ldots, t_{n}\right\} \in \mathrm{B}_{k}\left(\mathbf{F}_{\mathcal{V}}(X)\right)$. The base case follows exactly as in the proof of Theorem 2, as does the inductive step for the case where $\left\{t_{1}, \ldots, t_{n-1}, u v\right\} \in \mathrm{B}_{k+1}\left(\mathbf{F}_{\mathcal{V}}(X)\right)$ results from $\left\{t_{1}, \ldots, t_{n-1}, u\right\} \in \mathrm{B}_{k}\left(\mathbf{F}_{\mathcal{V}}(X)\right)$ and $\left\{t_{1}, \ldots, t_{n-1}, v\right\} \in \mathbf{B}_{k}\left(\mathbf{F}_{\mathcal{V}}(X)\right)$. Suppose now that $\left\{t_{1}, \ldots, t_{n-1}, u v\right\} \in \mathrm{B}_{k+1}\left(\mathbf{F}_{\mathcal{V}}(X)\right)$ follows from $\left\{t_{1}, \ldots, t_{n-1}, v u\right\} \in$ $\mathrm{B}_{k}\left(\mathbf{F}_{\mathcal{V}}(X)\right)$. By the induction hypothesis,

$$
\mathcal{K}_{\mathcal{V}} \models e \leq t_{1} \vee \cdots \vee t_{n-1} \vee v u
$$

and hence, since the quasi-equation $(e \leq x \vee y z) \Rightarrow(e \leq x \vee z y)$ is valid in all o-groups, $\mathcal{K}_{\mathcal{V}}=e \leq t_{1} \vee \cdots \vee t_{n-1} \vee u v$.

We prove (3) $\Rightarrow$ (1) by contraposition. Suppose that $\left\{t_{1}, \ldots, t_{n}\right\}$ extends to an order $\leq$ on $\mathbf{F}_{\mathcal{V}}(X)$. Then $t_{1}, \ldots, t_{n}$ are all negative with respect to the dual order $\leq{ }^{\partial}$ on $\mathbf{F}_{\mathcal{V}}(X)$. Let $\mathbf{L} \in \mathcal{K}_{\mathcal{V}}$ be the o-group with group reduct $\mathbf{F}_{\mathcal{V}}(X)$ and order $\leq^{\partial}$, and let $\varphi$ be the homomorphism from $\mathbf{T}^{\ell}(X)$ to $\mathbf{L}$ defined by mapping each $x \in X$ to $x \in F_{\mathcal{V}}(X)$. Then $\varphi(t)$ is $t \in F_{\mathcal{V}}(X)$ for each group term $t$, and, since $\leq^{\partial}$ is total, $\varphi\left(t_{1} \vee \cdots \vee t_{n}\right)=\varphi\left(t_{i}\right)=t_{i} \in F_{\mathcal{V}}(X)$ for some $i \in\{1, \ldots, n\}$. But given that $t_{1}, \ldots, t_{n}$ are all negative, also

$$
\varphi\left(t_{1} \vee \cdots \vee t_{n}\right)=t_{i}<^{\partial} e=\varphi(e) .
$$

So $\mathcal{K}_{\mathcal{V}} \not \models e \leq t_{1} \vee \cdots \vee t_{n}$.

Corollary 5. If $\mathbf{F}_{\mathcal{V}}(X)$ is orderable, then $S \subseteq F(X)$ extends to an order on $\mathbf{F}_{\mathcal{V}}(X)$ if and only if $\mathcal{K}_{\mathcal{V}} \forall=e \leq t_{1} \vee \cdots \vee t_{n}$ for all $\left\{t_{1}, \ldots, t_{n}\right\} \subseteq S$.

We may also view these results from the opposite direction, starting with some particular variety of $\ell$-groups rather than a variety of groups. Let $\mathcal{L}$ be a variety of representable $\ell$-groups defined relative to $\mathcal{R} \mathcal{G}$ by a set $\Sigma \subseteq(T(X))^{2}$ of group equations, and let $\mathcal{V}$ be the variety of groups defined by $\Sigma$. If the relatively free group $\mathbf{F}_{\mathcal{V}}(X)$ is orderable, then Theorem 8 implies that $\left\{t_{1}, \ldots, t_{n}\right\} \subseteq T(X)$ extends to an order on $\mathbf{F}_{\mathcal{V}}(X)$ if and only if $\mathcal{L} \not \models e \leq t_{1} \vee \cdots \vee t_{n}$. Note also that in this case, if an $\ell$-group equation fails in $\mathcal{L}$, then it fails in $\mathbf{F}_{\mathcal{V}}(X)$ equipped with some order. So, if the relatively free groups of $\mathcal{L}$ are orderable, then $\mathcal{L}$ is generated as a variety by the class of o-groups with group reducts of $\mathbf{F}_{\mathcal{V}}(X)$. In particular, the variety of representable $\ell$-groups is generated as a variety by the class of o-groups whose group reducts are free groups.

Finally, let us remark that some questions discussed in Section 3 for right orders and $\ell$-groups are still open for orders and representable $\ell$-groups. It follows 
from Theorem 8 that the decidability of the word problem for free representable $\ell$-groups is equivalent to the problem of deciding whether a given finite set of elements of a free group extends to an order. Both of these problems are, as far as we know, still open. Also from a topological perspective, much less is known in the case of orders. The topological space $\mathcal{B O}(\mathbf{G})$ of orders on a group $\mathrm{G}$ consists of the set of normal subsemigroups $P$ such that $P \cup P^{-1}=G \backslash\{e\}$, equipped with the induced powerset topology, and forms a closed subspace of $\mathcal{R O}(\mathbf{G})$. It is not known, however, whether the space $\mathcal{B O}(\mathbf{F}(k))$ of orders on a (non-trivial) finitely generated free group has any isolated point. Equivalently, it is not known if for $t_{1}, \ldots, t_{n} \in T(k)$, whenever $\mathcal{R G} \models e \leq t_{1} \vee \cdots \vee t_{n} \vee s$ or $\mathcal{R G}=e \leq$ $t_{1} \vee \cdots \vee t_{n} \vee s^{-1}$ for all $s \in T(k)$, then $\mathcal{R} \mathcal{G} \models e \leq t_{1} \vee \cdots \vee t_{n}$.

\section{References}

[1] R. Botto Mura and A. Rhemtulla. Orderable Groups, volume 27 of Lecture Notes in Pure and Applied Mathematics. M. Dekker, New York-Basel, 1977.

[2] M. Botur, J. Kühr, L. Liu, and C. Tsinakis. The Conrad program: from $\ell$-groups to algebras of logic. Journal of Algebra, 450:173-203, 2016.

[3] A. Clay and D. Rolfsen. Ordered Groups and Topology, volume 176 of Graduate Studies in Mathematics. American Mathematical Society, 2016.

[4] A. Clay and L. H. Smith. Corrigendum to: On ordering free groups, Journal of Symbolic Computation, 40(6):1285-1290, 2005. Journal of Symbolic Computation, 44(10):1529-1532, 2009.

[5] A. Colacito and V. Marra. Orders on groups, and spectral spaces of latticegroups. arXiv preprint:1901.07638, 2019.

[6] A. Colacito and G. Metcalfe. Proof theory and ordered groups. In Proceedings of WoLLIC 2017, volume 10388 of LNCS, pages 80-91. Springer, 2017.

[7] P. Conrad. Right-ordered groups. Michigan Mathematical Journal, 6(3):267-275, 1959.

[8] B. Deroin, A. Navas, and C. Rivas. Groups, orders, and dynamics. arXiv preprint: $1408.5805,2014$.

[9] L. Fuchs. Partially Ordered Algebraic Systems. Pergamon Press, 1963. 
[10] N. Galatos and G. Metcalfe. Proof theory for lattice-ordered groups. Annals of Pure and Applied Logic, 8(167):707-724, 2016.

[11] J. Gil-Férez, A. Ledda, F. Paoli, and C. Tsinakis. Projectable $\ell$-groups and algebras of logic: categorical and algebraic connections. Journal of Pure and Applied Algebra, 220(10):3514-3532, 2016.

[12] J. Gil-Férez, A. Ledda, and C. Tsinakis. Hulls of ordered algebras: Projectability, strong projectability and lateral completeness. Journal of Algebra, 483:429-474, 2017.

[13] W. C. Holland. The lattice-ordered group of automorphisms of an ordered set. Michigan Mathematical Journal, 10:399-408, 1963.

[14] W. C. Holland. The largest proper variety of lattice-ordered groups. Proceedings of the American Mathematical Society, 57:25-28, 1976.

[15] W. C. Holland and S. H. McCleary. Solvability of the word problem in free lattice-ordered groups. Houston Journal of Mathematics, 5(1):99-105, 1979.

[16] H. A. Hollister. Contributions To The Theory Of Partially Ordered Groups. $\mathrm{PhD}$ thesis, University of Michigan, 1965.

[17] D. Kielak. Groups with infinitely many ends are not fraction groups. Groups, Geometry and Dynamics, 9:317-323, 2015.

[18] V. M. Kopytov and N. Y. Medvedev. The Theory of Lattice-Ordered Groups. Kluwer, 1994.

[19] S. McCleary. Free lattice-ordered groups represented as o-2 transitive 1permutation groups. Transactions of the American Mathematical Society, 290:81-100, 1985.

[20] M. Ohnishi. Linear order on a group. Osaka Mathematical Journal, 4:17-18, 1952.

[21] A. S. Sikora. Topology on the spaces of orderings of groups. Bulletin of the London Mathematical Society 36(4): 519-526, 2004.

[22] D. Wessel. Ordering groups syntactically. Manuscript, 2017. 